\title{
T1rho shortening effect of fat in liver steatosis after fat suppression: approximate estimation in a methionine and choline-deficient (MCD) diet rat model
}

\author{
Feng Zhao ${ }^{1 \wedge}$, Nan Zhou ${ }^{2}$, Xiaoqi Wang ${ }^{3}$, Ji-Li Wang ${ }^{4}$, Wei-Xiang Zhong ${ }^{4}$, Min Deng ${ }^{5}$, Cun-Jing Zheng ${ }^{5}$, \\ Jian $\mathrm{He}^{2}$, Sen-Xiang Yan $^{1}$, Yì Xiáng J. Wáng ${ }^{5} \wedge$ \\ ${ }^{1}$ Department of Radiation Oncology, The First Affiliated Hospital, Zhejiang University School of Medicine, Hangzhou, China; ${ }^{2}$ Department \\ of Radiology, The Affiliated Drum Tower Hospital of Nanjing University Medical School, Nanjing, China; ${ }^{3}$ Philips Healthcare, Beijing, China; \\ ${ }^{4}$ Department of Pathology, The First Affiliated Hospital, Zhejiang University School of Medicine, Hangzhou, China; ${ }^{5}$ Department of Imaging and \\ Interventional Radiology, Faculty of Medicine, The Chinese University of Hong Kong, Shatin, Hong Kong SAR, China
}

Correspondence to: Dr. Yì Xiáng J. Wáng. Department of Imaging and Interventional Radiology, Faculty of Medicine, The Chinese University of Hong Kong, Shatin, Hong Kong SAR, China. Email: yixiang_wang@cuhk.edu.hk.

Submitted Nov 07, 2020. Accepted for publication Nov 16, 2020.

doi: 10.21037/qims-20-1253

View this article at: http://dx.doi.org/10.21037/qims-20-1253

In our recent study using a rat model of non-alcoholic fatty liver disease (NAFLD) induced with methionine and choline-deficient (MCD) diet, we demonstrated that collagen deposition in the liver strongly contribute to liver T1rho elongation, while fat deposition contributes to T1rho shortening $(1,2)$. However, there were two questions remain unanswered. The first is that how the fat content's contribution to T1rho shortening can be quantified. Secondly, since we estimated that $1 \%$ collagen area increase contributed to T1rho value increase of $1.35 \mathrm{~ms}$, and this observation might have been damped by that fat deposition always co-existed with collagen deposition in this animal model, thus it remains undetermined that $1 \%$ collagen increase contributes to how much T1rho value elongation in the absence of liver steatosis. Hereby we try to address these two questions.

Using last study's data of each experimental rat's collagen content (in percentage area measured with histology), MRI measured fat content (in percentage), and T1rho (in ms, millisecond), we ranked the results according to the collagen content and selected the 15 pairs where the collagen content difference within each pair was $\leq 0.03 \%$. Since liver collagen content of experimental rats measured from 0.34
$6.46 \%$ (mean \pm SD: $2.58 \% \pm 1.54 \%$ ), the within pair collagen content difference of these rats could be considered to be 'zero' (1). We took the assumption that, in our MCD model, inflammation's contribution to T1rho per se was minimal, and was instead mediated via collagen deposition. Thus, leaving aside the issue of measurement imprecision, we can hypothesize that difference between each pair's liver T1rho value was primarily due to the difference in liver fat content. Note we confirmed that liver fat content measured by MRI was almost the same as measured by histology (1). Figure $1 A$ shows, when disregarding the amount of fat content difference, higher liver fat content is seen with longer liver T1rho value in 5 pairs while with shorter T1rho value in 10 pairs. The mean difference among the 15 pairs was -1.028 (95\% confidence interval: $-1.9499,-0.1061, \mathrm{P}=0.031)$. The mean difference value of 10 pairs with T1rho shortening was three times of that of the 5 pairs with T1rho elongation $(-1.874 v s$. $0.664 \mathrm{~ms})$. A mean of $8.72 \%$ higher fat content was associated with a mean of $1.03 \mathrm{~ms}$ T1rho shortening (data from Figure 1A). A linear regression suggests 10\% fat increase contributed to $1.55 \mathrm{~ms}$ T1rho shortening (Figure 1B, Pearson $\mathrm{r}=-0.40$ ). We hypothesize that higher

^ ORCID: Feng Zhao, 0000-0002-4441-9992; Yì Xiáng J. Wáng, 0000-0001-5697-0717. 

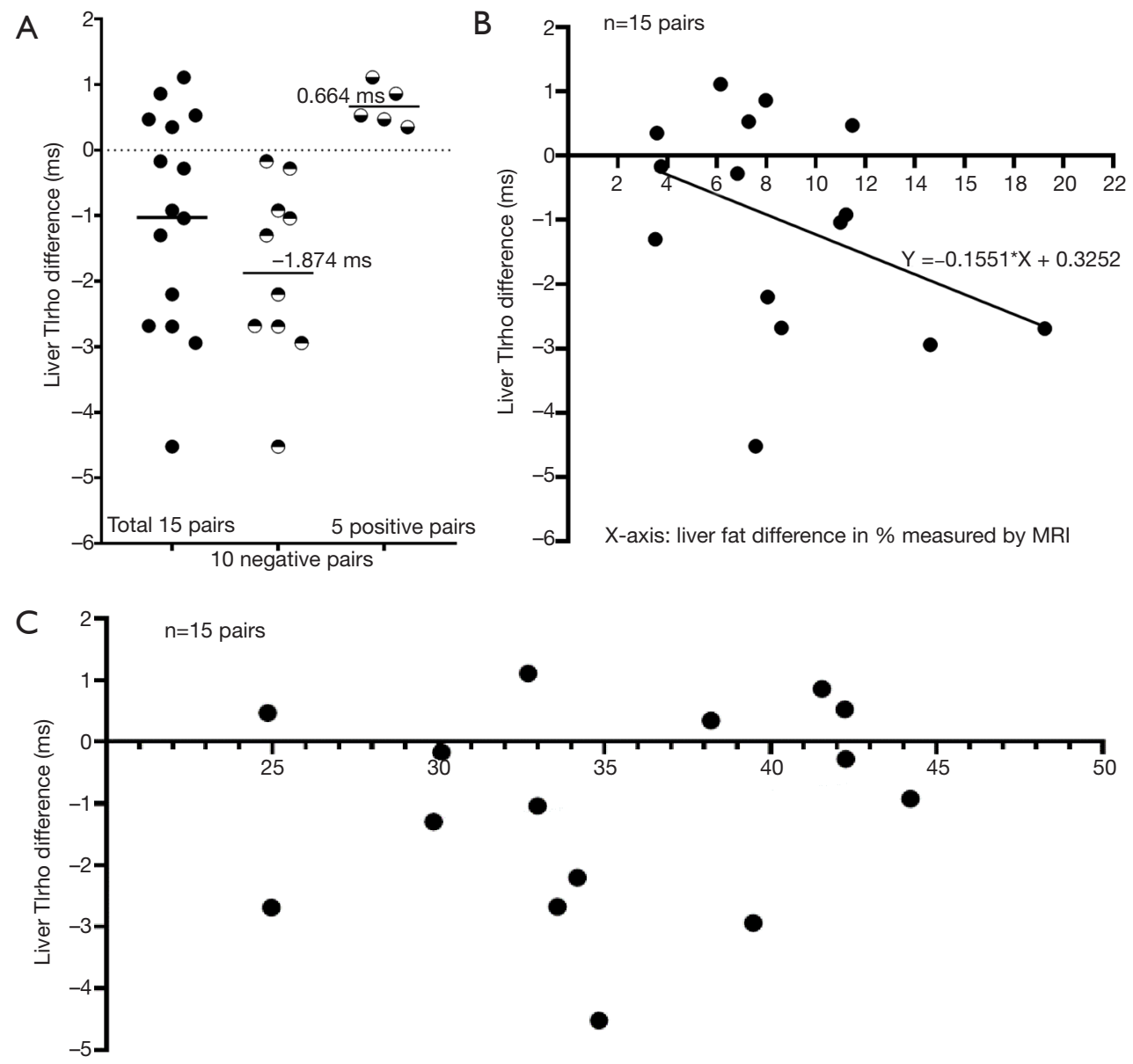

Liver fat content \% by MRI

Figure 1 A crude estimation of liver fat's contribution to T1rho shortening. (A) Higher liver fat content is with longer liver T1rho value in 5 pairs while with shorter T1rho value in 10 pairs. Y-axis: difference of liver T1rho for each pair (T1rho of liver with higher fat content minus T1rho of liver with lower fat content). One dot denotes one liver pair with same collagen content. Since the 15 pairs of livers had similar collagen content, the assumption is that if fat deposition has no impact on liver T1rho, then the T1rho difference of these 15 pairs should be all around zero. (B) Linear regression of the 15 liver pairs. (C) each pair's liver T1rho difference along the extent of total MRI measured liver fat content (tentatively suggest that fat's \% impact on T1rho shortening is not affected by the total \% fat in the liver, i.e., an increase from $25 \%$ to $30 \%$ fat may have the same T1rho shortening as an increase from $40 \%$ to $45 \%$ fat). bar in (A): mean.

liver fat content was seen with higher liver T1rho value in 5 pairs was due to measurement imprecision for collagen content and/or T1rho.

If the estimation is correct that $10 \%$ fat increase contributes to $1.55 \mathrm{~ms}$ T1rho shortening, we can hypothesize that the correlation of liver collagen $v s$. liver T1rho in experimental rats would be improved if we correct the liver T1rho by:

$$
\text { corrected T1rbo }=\text { measured T1rho }+(0.155 \times ? \% \text { fat })
$$

For example, if the liver fat is $25 \%$, then the equation will be corrected T1rho $=$ measured T1rbo $+(0.155 \times 25)$.

The liver T1rho data of all experimental rats with and without fat correction is shown in Figure 2. The original Pearson $r$ was 0.8157 . This $r$ was improved to 0.8374 when the corrected T1rho values were used. Due to the limited sample size for the analysis in Figure 1, this coefficient of 1.55 may not be optimal, we further hypothesize that the correction coefficient achieves the best Pearson $r$ would be more precise. We tested correction coefficient of 1.9 , $1.7,1.55,1.5,1.3,1.1,1,0.9,0.8$, and 0.7 and found that the coefficient of 0.9 offered the best Pearson $r$ of 0.8447 . 
However, we noted that this was to better accommodate the initial lowest four T1rho values (Figure 2). If we removed these lowest four T1rho values, then indeed coefficient of 1.55 or 1.6 offered best Pearson $\mathrm{r}$ of 0.8586 . It can also be appreciated visually that fat correction improved the fitting (Figure 2). Thus, we tentatively accept that corrected T1rho= measured T1rho $+(0.155 \times$ ? \% fat $)$ is a good approximate for

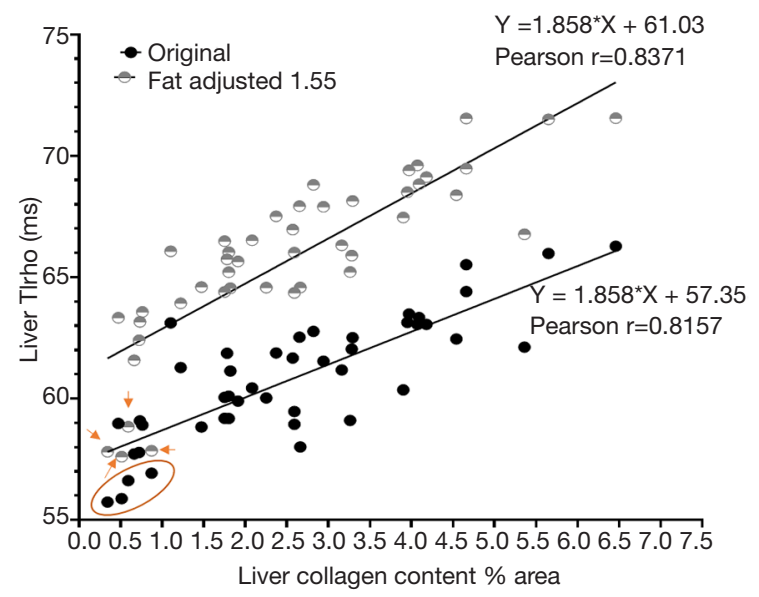

Figure 2 The correlation of liver collagen (measured by histology) and liver T1rho data of all experimental rats $(n=45)$ with (fat adjusted 1.55) and without fat correction (original). There are 4 outliers with low liver collagen content and very low liver T1rho (oval circle and arrows). our data.

The above discussion opens a possibility that the relationship between liver collagen content and T1rho increase may not be linear. If we arbitrarily separated the rats into two groups, i.e., those with $<1 \%$ liver collagen content and those with $\geq 1 \%$ liver collagen content, it can be shown that those with liver collagen content $<1 \%$ had a much steeper increasing slope for the relationship between liver collagen and T1rho (Figure 3). After correction with liver fat, it can be estimated that, for those livers with $\geq 1 \%$ liver collagen, $1 \%$ additional collagen contributes to $1.3 \mathrm{~ms}$ liver T1rho elongation. Non-linear fitting for our total 53 rats' data suggests the relationship between liver collagen content and fat corrected T1rho can be represented according to Eq. [2] (Figure 4):

$$
y=5.0308^{*} \ln (x)+61.88
$$

where $\mathrm{x}$ is the collagen content and $\mathrm{y}$ is the liver T1rho after fat correction. The higher goodness-of-fit shown in Figure $4 B$ than in Figure $4 A$ further support fat correction and that collagen is the dominant contributor for T1rho elongation in this animal model (1). For the data of livers with $<1 \%$ liver collagen, the results here should be interpreted with caution. Low liver collagen content may be more difficult to measure accurately, and this phenomenon may be only related to this animal model. Thus, the equation-2 should be considered as 'hypothesis generating' only.
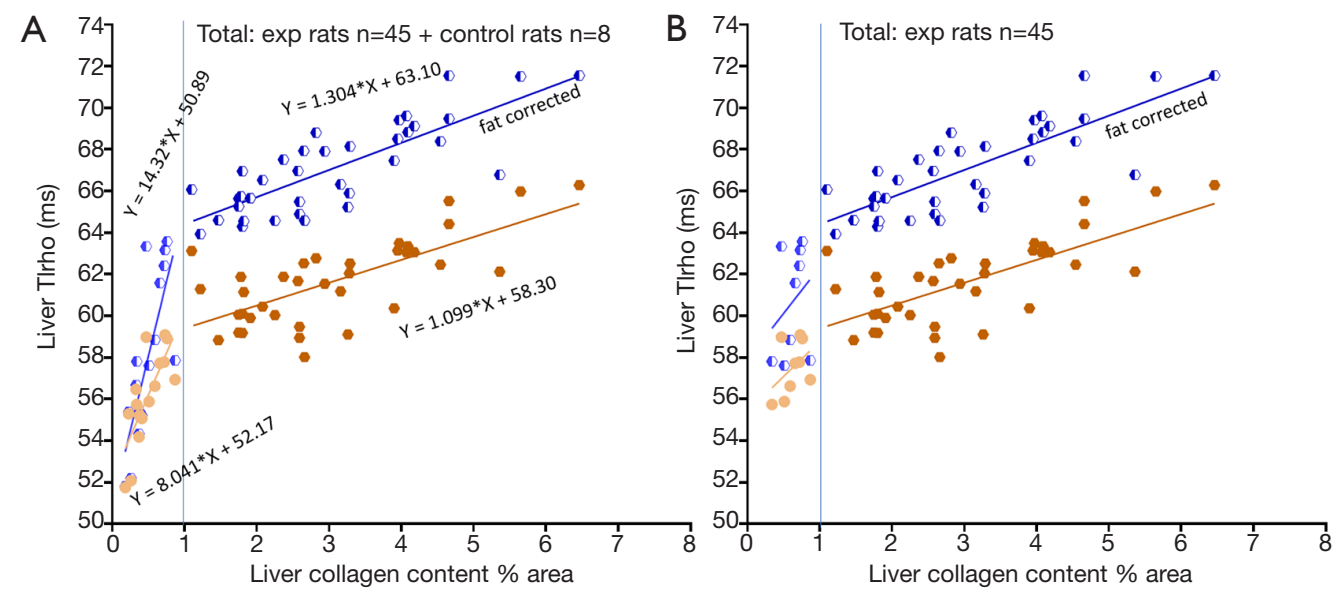

Figure 3 The relationship between liver collagen content (measured by histology in percent area) and liver T1rho with (blue semi-solid dots) or without (orange solid dots) fat contribution correction. Rats are separated into two groups, those with $<1 \%$ liver collagen content and those with $\geq 1 \%$ liver collagen content. For liver with $\geq 1 \%$ liver collagen, the Pearson $\mathrm{r}$ is 0.705 for original T1rho measure, while 0.803 for T1rho measure with fat correction (both $\mathrm{P}<0.0001$ ). (A) The results of 45 experimental rats and 8 control rats. (B) The results of only 45 experimental rats. 
A

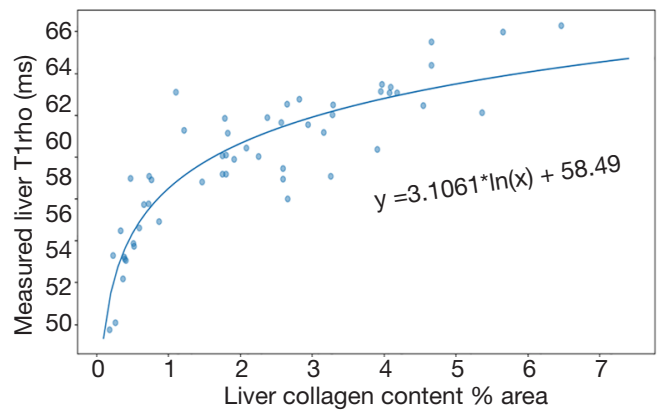

B

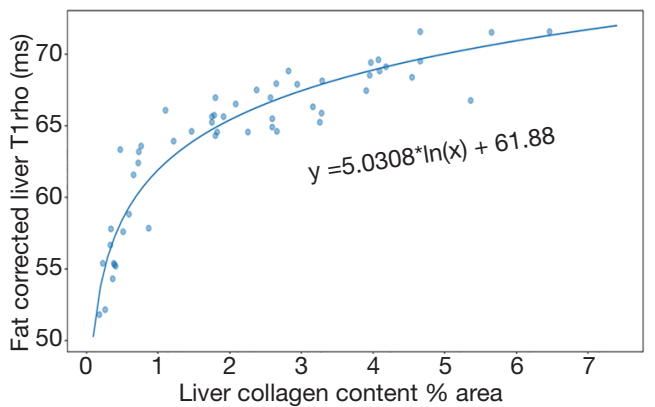

Figure 4 The relationship between liver collagen content (measured by histology in percent area) and liver T1rho fitted with an exponential growth curve. (A) Without fat contribution correction; (B) with fat contribution correction. It can be visually appreciated that the goodnessof-fit is better in (B) than in (A). $\mathrm{n}=45$ experimental rats \& 8 control rats.
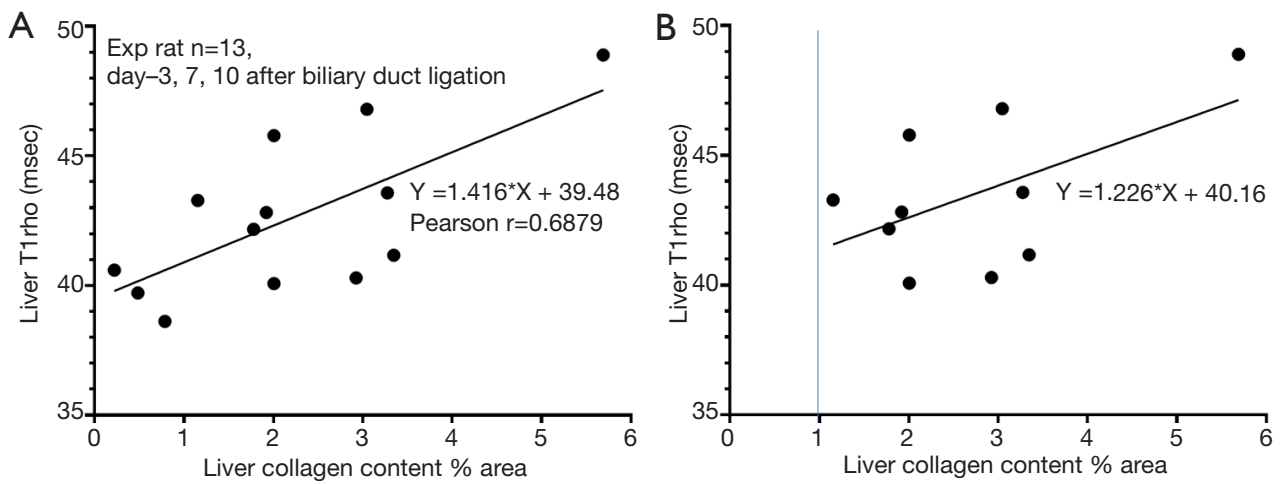

Figure 5 The relationship between liver T1rho value and liver collagen content (measured by histomorphometry) in experimental rats with biliary duct ligation. (A) All 13 rats. (B) 10 rats with liver collagen content >1\% area. Data were from (3). Reused with permission.

In order to validate the analysis presented above, we reanalyzed our previous results using a biliary duct ligation model (3). Thirteen experimental rats had both liver T1rho and liver collagen histomorphometry measurements available for analysis. The results show with all 13 rats, $1 \%$ collagen increase contributed to 1.4 ms liver T1rho elongation (Figure $5 A$ ). If the three rats with liver collagen less than $1 \%$ were removed, then $1 \%$ additional collagen contributed to $1.2 \mathrm{~ms}$ liver T1rho elongation (Figure 5B). While due to the small sample size, we should interpret the data in Figure 5 with caution, the data do seem to further confirm that, in the absence of liver steatosis, $1 \%$ collagen increase contributes to $1.2-1.4 \mathrm{~ms}$ liver T1rho elongation.

Based on the analysis above, there is the possibility that a $10 \%$ increase of fat deposition in a liver (e.g., an increase from a normal $2 \%$ liver fat to a steatosis status of $12 \%$ liver) would cancel out the T1rho elongation contribution by $1.1 \%$ increase of collagen deposition (e.g., an increase from a normal $0.3 \%$ liver collagen to early stage-1 fibrosis of $1.4 \%$ liver collagen). Note in reference-1, stage- 1 fibrosis had liver collagen content ranging from $1.1-3.26 \%$ with a median value of $1.8 \%$ ). In our MCD model study, we had 5 experimental rats graded as did not have inflammation and did not have fibrosis (1). These 5 experimental rats all had stage-1 steatosis, with a mean MRI measured liver fat of $17.38 \%$ (range: $5.98-30.0 \%$ ), while the 8 control rats had a mean MRI measured liver fat of $0.89 \%$ (range: $0.38-1.33 \%$ ). Quantitative histomorphometry did initially show 4 of these rats had slightly increased collagen deposition. Since the liver steatosis might have damped T1rho measure, the equation-1 was tentatively used to correct T1rho value both for control rats and the 4 experimental rats. The results show the corrected T1rho value allow better separation of control rats and experimental rats (Figure 6).

Of note, during the study rat No. 10 was initially histomorphometrically measured to have collagen of 

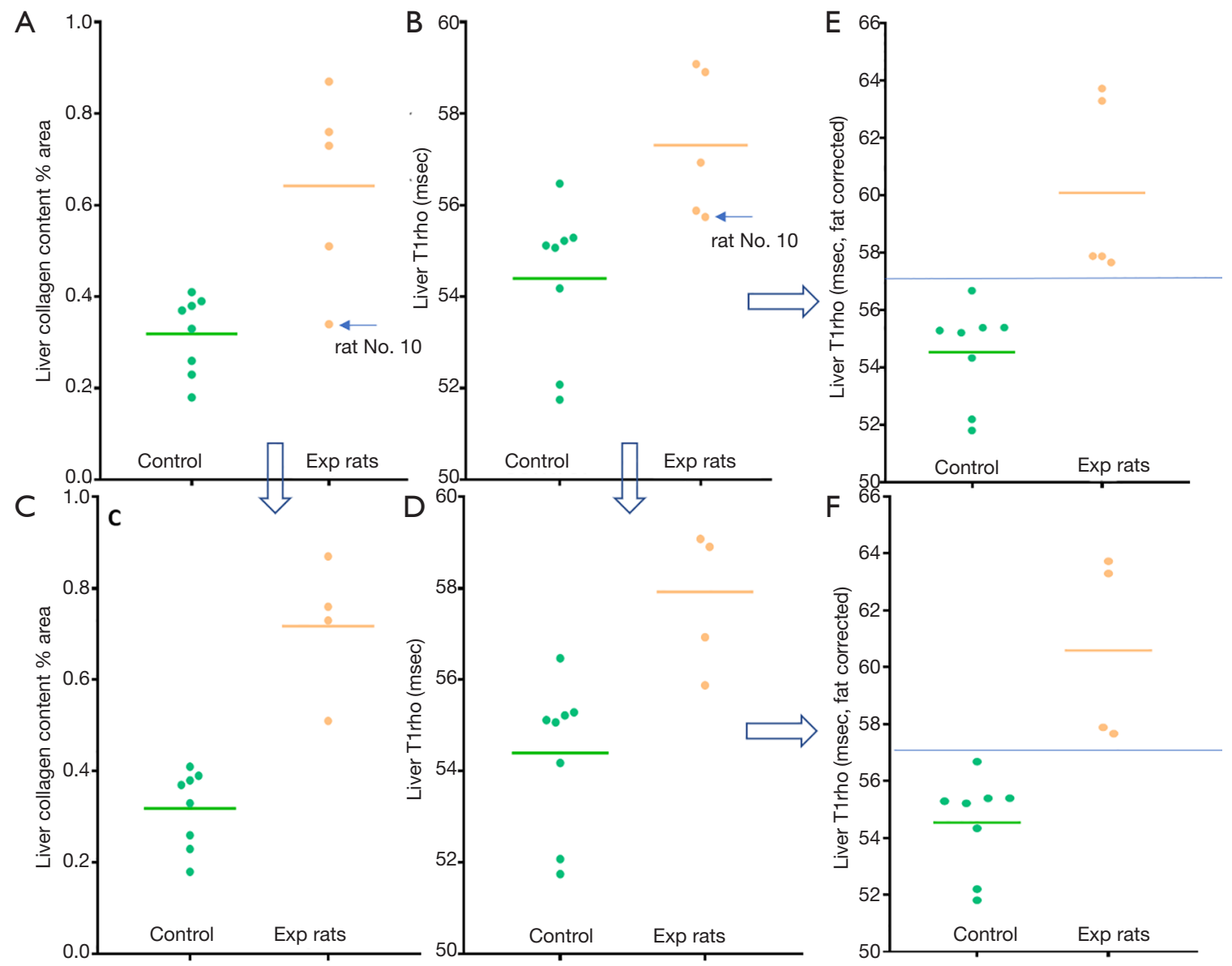

Figure 6 Liver T1rho with fat contribution correction allows complete separation of control rats ( $\mathrm{n}=8$ ) and five experiment rats without positive inflammation and without fibrosis grading. (A) Liver collagen of control rats and experimental rats. (B) Liver T1rho of control rats and experimental rats, showing partial overlap between these two groups of rats. After removing experimental rat No.10, (A) becomes (C), and (B) becomes (D) (arrow). After liver T1rho is adjusted with equation-1, (B) becomes (E), and (D) becomes (F) (arrow). In both (E) and (F), adjusted liver T1rho allows complete separation of control rats $(\mathrm{n}=8)$ and five experiment rats fed with MCD. Experimental Rat No. 10 had initial collagen measure similar to control rat, while after adjusted liver T1rho, it is separated from control rats by a longer T1rho measure. This may constitute a false positive result after T1rho correction with fat contribution. One study pathologist was contacted and asked to remeasure this rat's collagen (without be informed why a re-measurement was requested). The result came out the second measure was $0.52 \%$, thus higher than the control rats. bar: mean.

$0.34 \%$. Considering the MRI analysis in Figure 6, the collagen content was re-measured after the publication of reference- 1 , and the result turned out to be $0.52 \%$, which agrees with the liver T1rho feature of this rat. While due to the potential sampling error of histomorphometry, the liver collagen content ground truth for this rat remains unknown, our analysis raised the possibility that liver T1rho has the potential to pinpoint errors of histomorphometry measure (the initial liver collagen measure of rat No. 10 was indeed a outlier from other 4 experimental rats which measured higher liver collagen content than the control rats). However, it should be noted that whether equation-1 is valid at very low concentration of liver collagen remains to be confirmed. There is a theorical risk that this correction may lead to over diagnosis of liver fibrosis. Further studies shall clarify this point.

We hereby attempt to explain the mechanism of T1rho shortening effect of fat under fat suppression. A fat saturation method usually cannot fully suppress fat signal in liver and other fatty tissues, due to the variations of T1 
of fatty tissues and also due to the imperfections of $B_{1}$ and $\mathrm{B}_{0}$. After the initial $90^{\circ}$ pulse of the T1rho preparation, residual fat proton spins are flipped to the transverse plane along with water proton spins, but will move in the rotation frame with a period of $2.3 \mathrm{~ms}$ (3T) which is substantially shorter than the spin lock preparation duration. The fat proton spins are then turned into Z-direction in one half period, and turned into the opposite direction in another half period. Eventually this residual fat signal will decrease to zero under the effect of spin lock pulse. So residual fat proton spins shall not have significant impact on T1rho measurement. However, as shown in Figure S1, after the saturation pulse and the first excitation pulse which flips water and the residual fat proton spins $\left(M_{o}\right)$ to transverse plane $\left(M_{x y}\right)$, the water and fat longitudinal signals $\left(M^{\mathrm{w} 1}{ }_{0}\right.$, $\mathrm{M}^{\mathrm{f1}}{ }_{0}$ ) grow due to $\mathrm{T} 1$ relaxation during the spin-lock period. The gradually growing water signal in longitude direction $\left(\mathrm{M}^{\mathrm{w1}}{ }_{0}\right)$ is continually flipped by the spin-lock pulse, and its precession $\left(\mathrm{M}^{\mathrm{w} 1}{ }_{\mathrm{xy}}\right)$ is in the plane perpendicular to the spinlock pulse, and is then also perpendicular to the transverse signal under spin-lock process $\left(M_{x y}\right)$. The growth rate of spins in the longitude direction is constant and is inversely proportional to $\mathrm{T} 1$ value and fat signal grows faster than water signal. However, the fat proton spins $\left(\mathrm{M}^{\mathrm{f} 1}{ }_{\mathrm{xy}}\right)$ flipped into transverse plane undergo procession slower than water proton $\left(\mathrm{M}_{\mathrm{xy}}^{\mathrm{w} 1}\right)$ and are not stable in ration frame. The dynamics of fat signal recover $\left(\mathrm{M}^{\mathrm{fl}}{ }_{0}\right)$ in longitude direction, flip $\left(\mathrm{M}_{\mathrm{xy}}^{\mathrm{f1}}\right)$ by spin lock pulse and moving in the rationing frame eventually accumulates fat signal $\left(\mathrm{M}^{\mathrm{f} 1}{ }_{\mathrm{xy}}\right)$ opposite to the spins under spin locking $\left(M_{x y}\right)$, and reduces the total signal acquired afterwards. More fat content leads to stronger signal reduction; and this reduces T1rho in this type of measurement.

In conclusion, the additional analysis presented here further confirms that liver fat deposition contributes to T1rho shortening in the rat NAFLD model. For those with $\geq 1 \%$ liver collagen, an approximately corrected T1rbo=measured T1rbo+ $(0.155 \times$ ? \% fat $)$ improves the correlation between liver collagen content and liver T1rho. However, while the concepts presented here may be correct, the quantifications should be regarded as crude approximation.

\section{Acknowledgments}

Funding: This study was supported by the National Nature Science Foundation of China (81701683), Hong Kong GRF grants (Project No. 14109218).

\section{Footnote}

Provenance and Peer Review: This article was commissioned by the editorial office, Quantitative Imaging in Medicine and Surgery. The article did not undergo external peer review.

Conflicts of Interest: All authors have completed the ICMJE uniform disclosure form (available at http://dx.doi. org/10.21037/qims-20-1253). YXJW serves as an unpaid Editor-in-Chief of Quantitative Imaging in Medicine and Surgery. XW is an employee of Philips Healthcare. The other author has no conflicts of interest to declare.

Open Access Statement: This is an Open Access article distributed in accordance with the Creative Commons Attribution-NonCommercial-NoDerivs 4.0 International License (CC BY-NC-ND 4.0), which permits the noncommercial replication and distribution of the article with the strict proviso that no changes or edits are made and the original work is properly cited (including links to both the formal publication through the relevant DOI and the license). See: https://creativecommons.org/licenses/by-nc-nd/4.0/.

\section{References}

1. Zhao F, Zhou N, Wang JL, Zhou H, Zou LQ, Zhong WX, He J, Zheng CJ, Yan SX, Wáng YXJ. Collagen deposition in the liver is strongly and positively associated T1rho elongation while fat deposition is associated with T1rho shortening: an experimental study of methionine and choline-deficient (MCD) diet rat model. Quant Imaging Med Surg 2020;10:2307-21.

2. Hectors SJ. Is MRI relaxometry parameter $T 1 \rho$ specific to fibrosis or confounded by concomitant pathological features? Quant Imaging Med Surg 2020;10:2408-10.

3. Koon CM, Zhang X, Chen W, Chu ES, San Lau CB, Wáng YX. Black blood T1rho MR imaging may diagnose early stage liver fibrosis: a proof-of-principle study with rat biliary duct ligation model. Quant Imaging Med Surg 2016;6:353-63.

Cite this article as: Zhao F, Zhou N, Wang X, Wang JL, Zhong WX, Deng M, Zheng CJ, He J, Yan SX, Wáng YX. T1rho shortening effect of fat in liver steatosis after fat suppression: approximate estimation in a methionine and choline-deficient (MCD) diet rat model. Quant Imaging Med Surg 2021;11(2):870-875. doi: 10.21037/qims-20-1253 


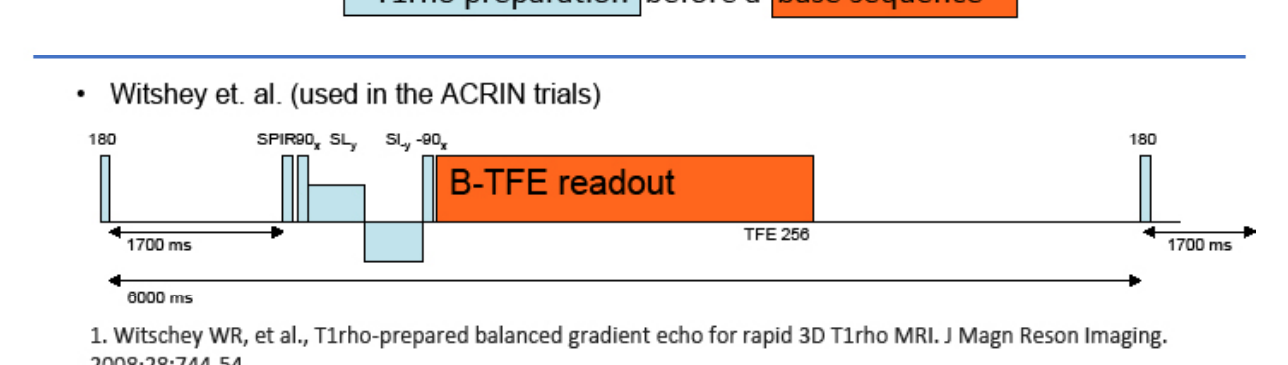

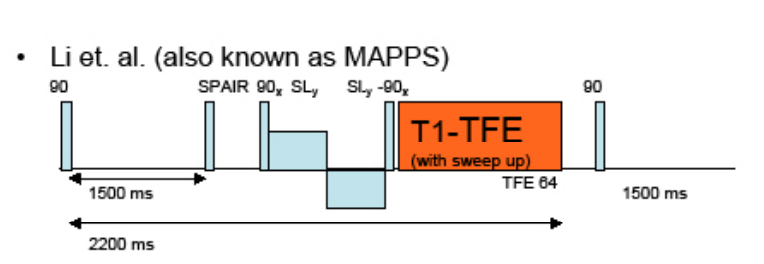
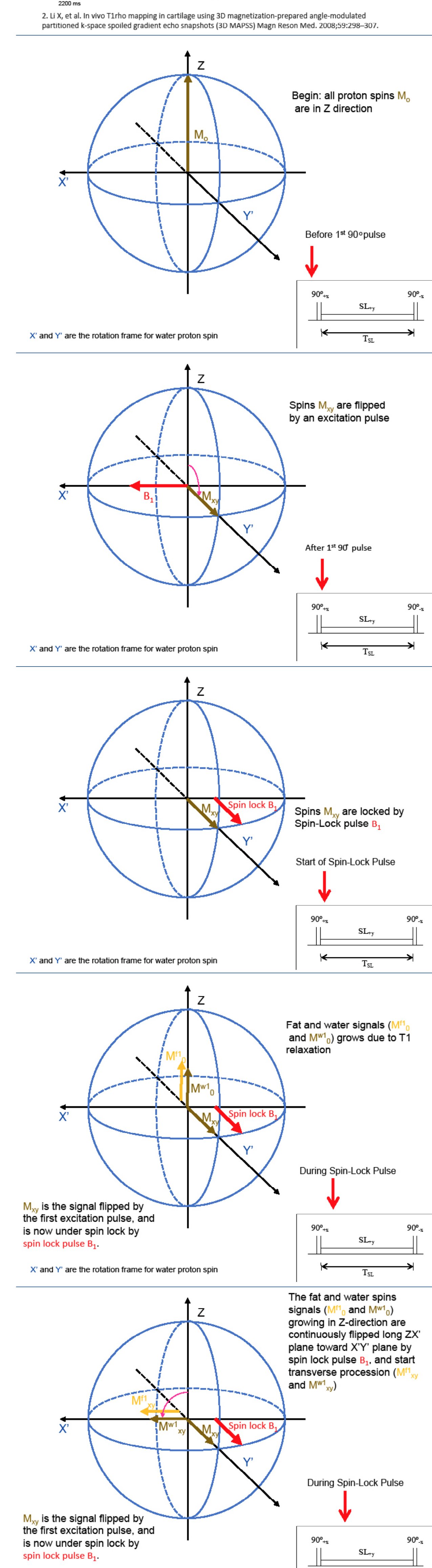

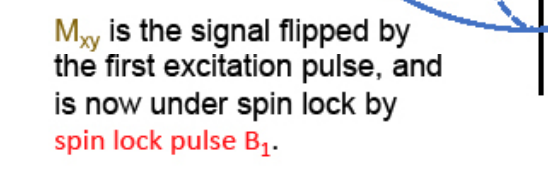

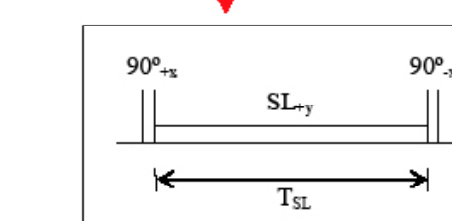
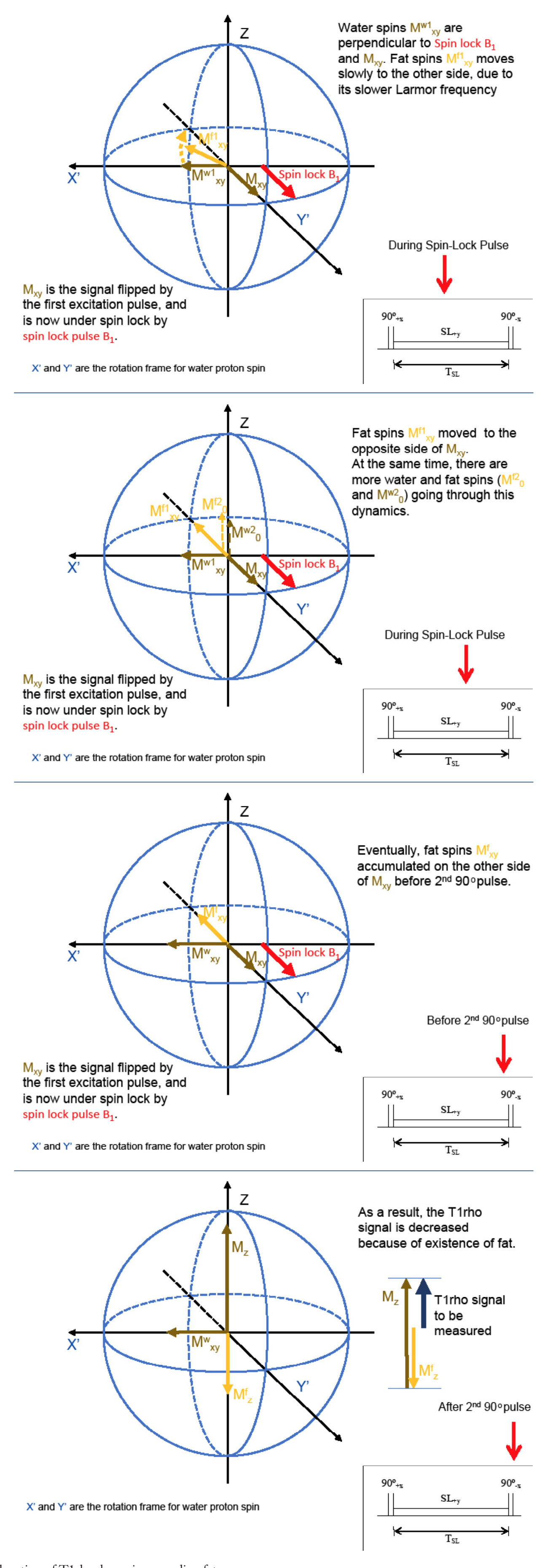